\title{
Erratum to: Brain structure differences among male schizophrenic patients with history of serious violent acts: an MRI voxel-based morphometric study
}

Noriomi Kuroki ${ }^{1,2,3^{*}}$, Hiroko Kashiwagi ${ }^{1}$, Miho Ota ${ }^{4}$, Masanori Ishikawa ${ }^{5}$, Hiroshi Kunugi ${ }^{4}$, Noriko Sato ${ }^{6}$, Naotsugu Hirabayashi ${ }^{1}$ and Toshio Ota ${ }^{2}$

\section{Erratum}

This article [1] has been updated to correct the image order. In the original article, Figs. 2 and 3 were interchanged. The figures are now in the correct position. This mistake was carried forward by production, and thus is not the fault of any authors of the manuscript.

\begin{abstract}
Author details
'Department of Psychiatry, National Center Hospital, National Center of Neurology and Psychiatry, 4-1-1 Ogawa-Higashi, Kodaira, Tokyo 187-8551, Japan. ${ }^{2}$ Department of Psychiatry, Saitama Medical University, 38 Morohongo Moroyama-machi, Iruma-gun, Saitama 350-0495, Japan. ${ }^{3}$ Department of Psychiatry, Tokyo Metropolitan Matsuzawa Hospital, 2-1-1 Kamikitazawa, Setagaya-ku, Tokyo 156-0057, Japan. ${ }^{4}$ Department of Mental Disorder Research, National Institute of Neuroscience, National Center of Neurology and Psychiatry, 4-1-1 Ogawa-Higashi, Kodaira, Tokyo 187-8502, Japan. ${ }^{5}$ Department of Social Welfare Services, Faculty of Human Science, Mejiro University, 4-31-1 Nakaochiai, Shinjuku-ku, Tokyo 161-8539, Japan. ${ }^{6}$ Department of Radiology, National Center Hospital, National Center of Neurology and Psychiatry, 4-1-1 Ogawa-Higashi, Kodaira, Tokyo 187-8551, Japan.
\end{abstract}

Received: 30 March 2017 Accepted: 4 April 2017

Published online: 12 April 2017

\section{Reference}

1. Noriomi K, Hiroko K, Miho O, Masanori I, Hiroshi K, Noriko S, Naotsugu H, Toshio O. Brain structure differences among male schizophrenic patients with history of serious violent acts: an MRI voxel-based morphometric study. BMC Psychiatry. 2017;17:105. doi:10.1186/s12888-017-1263-9.

\footnotetext{
* Correspondence: nkuroki-tky@umin.ac.jp

'Department of Psychiatry, National Center Hospital, National Center of Neurology and Psychiatry, 4-1-1 Ogawa-Higashi, Kodaira, Tokyo 187-8551, Japan ${ }^{2}$ Department of Psychiatry, Saitama Medical University, 38 Morohongo Moroyama-machi, Iruma-gun, Saitama 350-0495, Japan
} 